\title{
Determination of the profile of the worm-type tool
}

\author{
Aleksandr Golovko ${ }^{1, *}$ \\ ${ }^{1}$ Kazan Federal University, pr.Syuyumbike, d.10A, Naberezhnye Chelny, 423812 ,Russian Federation
}

\begin{abstract}
The cutting wedge of disk shaver at gear shaving has not fully satisfactory geometry: clearance The cutting wedge of the disk shaver when gear shaving is not fully geometrically satisfactory. The clearance on cutting edges are practically equal to zero. We would suggest this is the reason that the disk shavers machining of gear-wheels with large allowance is not possible. The construction of a worm-type tool is known also for clean machining of points of involute of gear-wheels made with continuous spiral cutting edges. The difference of this construction from the construction of $\mathrm{N}$. Chemborisov is that a cutting wedge has the structurally made clearance $\alpha$ (Authors: N. Smorkalov, V.Skripin, V.Ptitcin, IU.Belugin). Polishing of side of coil is the most responsible and labor intensive operation when making a worm-type tool with the continuous spiral cutting edges of construction of N.Smorkalov and other.Dependences allowing to define the coordinates of key points of axial section of side of coil of the worm-type tool were obtained. These coordinates will allow to define the parameters of setting of abrasive disc in relation to the worm-type tool.
\end{abstract}

One of the variants of the machining of gear teeth, used in engineering, involves the use of gear shaving as a semi-finishing operation before thermal machining and gear honing [1-5].

Gear shaving pertains to the methods of machining on the principle of so-called "free" rolling, in which there is no rigid kinematic connection between the tool and the gear wheel. This feature of the pro gear shaving process positively influences its dynamics, since there are no additional sources of vibrations inherent in the chain of rolling. At the same time, the absence of a rigid kinematic connection between the tool and the wheel leads to a significant decrease in the radial runout of the gear ring, the difference in adjacent circumferential steps, the accumulated error of the circumferential pitch at the angle of $180^{\circ}$, no oscillation of the measuring interaxial distance after the gear shaving occurs [6-13].

The cutting wedge of the disk shaver during gear shaving has a not quite satisfactory geometry: the clearance angels on the cutting edges are almost equal to zero. For this reason, the machining of gear wheels by rotary gear shaving tool with large allowances for machining (for example, after pre-rolling the teeth) is impossible. It should also be taken into account the high laboriousness of making gear shaving tools, the piecetime for gear grinding reaches $5 \ldots 6$ hours.

For clean treatment of points of gear-wheels can be used multishank tools allowing to use technology of Power Skiving (Fig. 1).

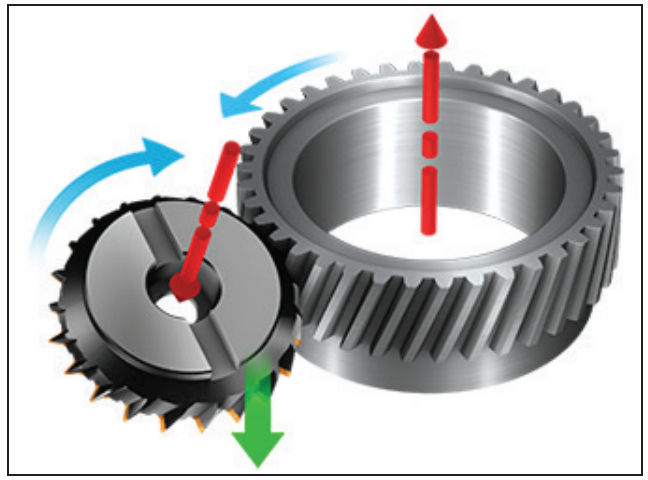

Fig. 1. Scheme of technology of Power Skiving.

However for using this technology the special expensive equipment is needed.

There are known the design of tools for finishing the teeth of involute gears with spiral-screw cutting edges made on one side of the turns $[14,15]$. The gears are machined with the coordinated rotation of the tool and the workpiece around their axes and moving them relative to each other in the axial direction of the workpiece, the processing of the right and left side of the profile of the teeth possible only after the wheel is reinstalled.

The drawback of these tools is the complexity of the design and manufacturing technology. A worm-type tool for finishing involute gear teeth is known with a continuous helical cutting edge, while its helical surface has a rectangular profile [16] [Fig. 2].

\footnotetext{
* Corresponding author: a.n.gol@yandex.ru
} 


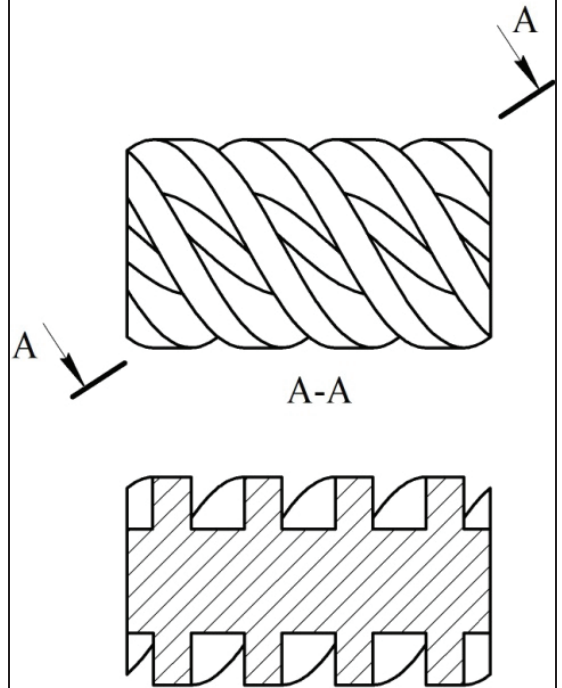

Fig. 2. Construction of the worm-type tool with spiral cutting edges (Authors: N. Chemborisov, R. Khisamutdinov, V. Avdeev).

The disadvantage of this tool is that the cutting wedge during its operation has a not quite satisfactory geometry: the clearance at the cutting edges is practically zero.

Also known is a worm-type tool for finishing the teeth of involute gears made with continuous screw cutting edges, different from the design of $\mathrm{N}$. Chemborisov and others [16] in that the cutting wedge has a structurally executed rear angle $\alpha$ [17] (Fig. 3).

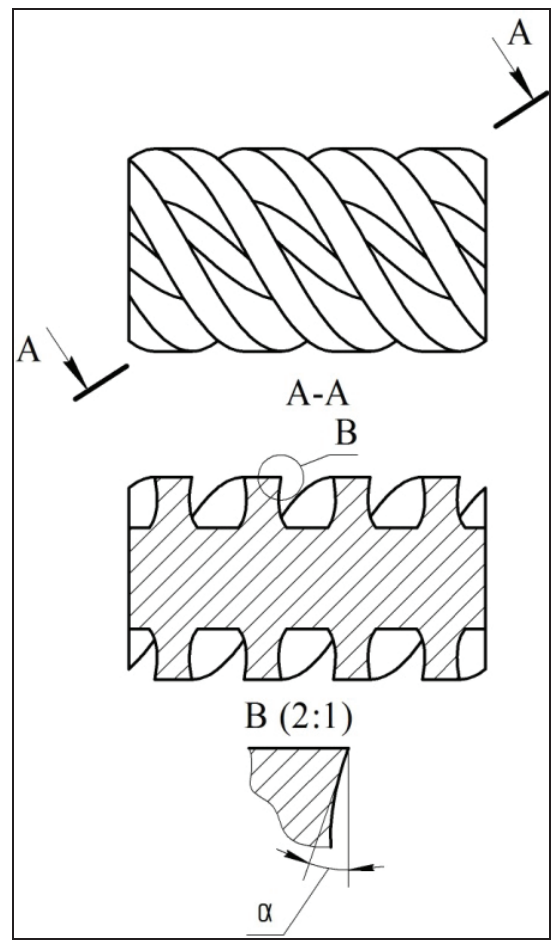

Fig. 3. Construction of a worm-type tool with spiral cutting edges (Authors: N. Smorkalov, V. Skripin, V. Ptitcin, IU. Belugin).

The machining is carried out with the coordinated rotation of the tool 1 and the wheel 2 (Fig.4,5,6,7).

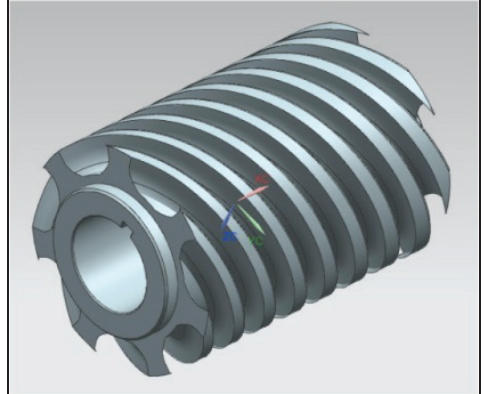

Fig.4. 3D model of a worm-type tool.

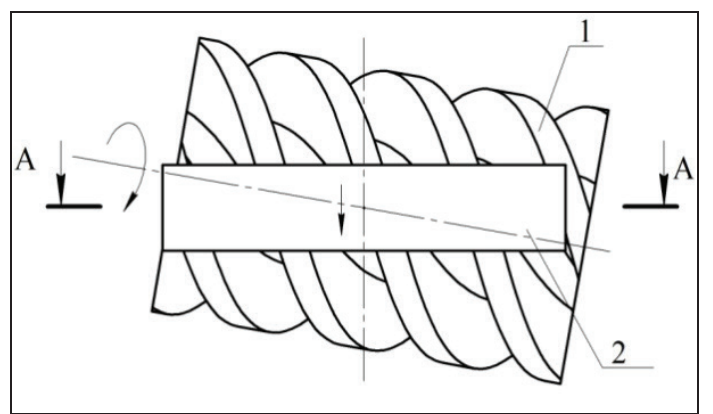

Fig. 5. Scheme of work of a worm-type tool with spiral cutting edges.

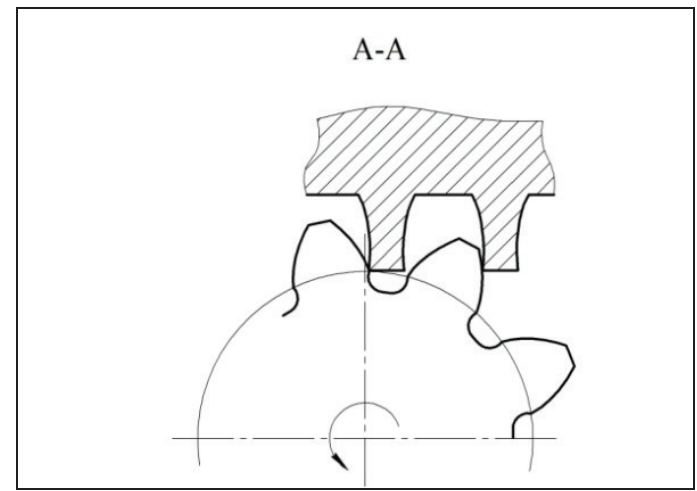

Fig. 6. Scheme of work of a worm-type tool with spiral cutting edges.

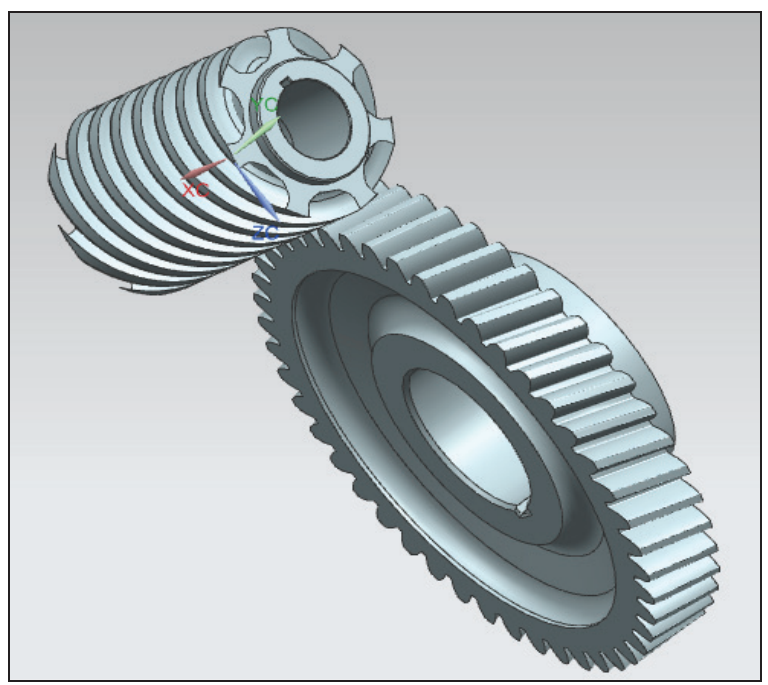

Fig.7. Visualization of adjustment of the worm-type tool on the machine in the module "Assembly" of the NX system. 
The cutting wedge element has a front surface coinciding with the outer surface of the tool and moves in relative motion from the head of the wheel to the leg.

The most important and time-consuming operation for the production of a worm-type tool with solid screw cutting edges is the grinding of the lateral surface of the winding. The lateral surface profile is specified initially in a section perpendicular to the screw cutting edge (Fig. 8).

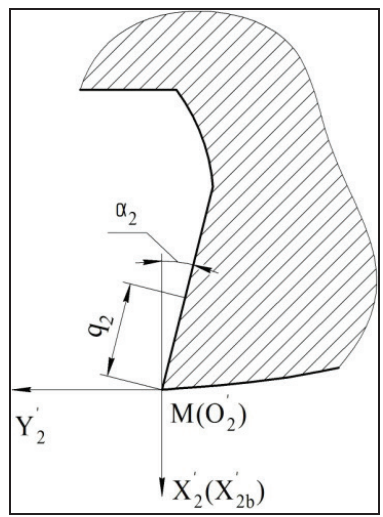

Fig. 8. The normal section of the profile coil of the worm-type tool.

The working section of the profile, which acts as the back surface of the cutting wedge, is formed in the form of a straight line segment. It is convenient to construct the solution of the inverse problem by comparing a theoretically accurate profile in the axial section and a profile obtained by numerical simulation.

This method is the most acceptable, since the use of a combination of the axial section of the worm-type tool and the cross-sections of the rotation surfaces (in this case cylindrical surfaces) considered in [18] makes it possible to obtain the $\mathrm{L}_{\mathrm{s}}$ (line of shaping) in the form of a straight line parallel to the axis of the worm tool type. In order to implement this scheme, it is necessary at the first stage to develop a methodology for calculating the coordinates of the nodal points of the profile of the lateral surface of the screw of the worm-type tool in the axial section.

We introduce a coordinate system $X_{2} Y_{2} Z_{2}$ rigidly connected with a worm-type tool (Fig. 9), whose axis $Z_{2}$ coincides with the axis $X_{2}$ of the worm-type tool, and the initial point of the screw cutting edge is located on the axis. Index 2 of the specified coordinate system is related to the fact that the worm-type tool in this case serves as a detail. With the current point $M$ of the screw cutting edge, we connect the origin of the coordinate system, $X_{2}^{\prime} Y_{2}^{\prime} Z_{2}^{\prime}$ in which we describe a straight section of the profile of the lateral surface of a worm-type tool. The axis $Z_{2}^{\prime}$ of the coordinate system coincides with the tangent to the helical cutting edge and makes an angle $\lambda_{2}$ with the end plane of the worm-type tool. The angle of rise of the screw cutting edge can be calculated using the well-known expression:

$$
\operatorname{tg} \lambda_{2}=\frac{p_{z 2}}{2 \pi r_{a 2}}
$$

Where $p_{z 2}$ - the axial movement of the helical cutting edge of the worm-type tool,

$r_{a 2}$ - the radius of the circumference of the wormtype tool protrusions.

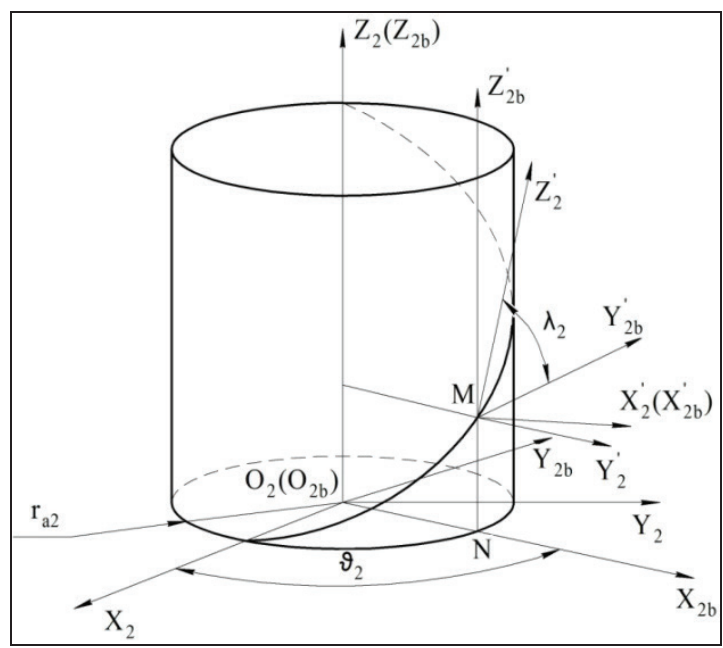

Fig. 9. Transition scheme from the system $X_{2}^{\prime} Y_{2}^{\prime} Z_{2}^{\prime}$ to the system $\mathrm{X}_{2} \mathrm{Y}_{2} \mathrm{Z}_{2}$.

The coordinate axis $X_{2}^{\prime}$ lies in the end plane of the worm-type tool and crosses its axis. Thus, the coordinate plane $X_{2}^{\prime} O_{2}^{\prime} Y_{2}^{\prime}$ coincides with the normal cross section. Equations of the rectilinear portion of the lateral surface of the coil of the worm-type tool can be written in the following form (Fig. 8):

$$
\begin{aligned}
& x_{2}^{\prime}=-q_{2} \cos \alpha_{2} \\
& y_{2}^{\prime}=-q_{2} \sin \alpha_{2}, \\
& z_{2}^{\prime}=0
\end{aligned}
$$

Where $q_{2}$ - the parameter determining the position of the point under consideration on the generator,

$\alpha_{2}$ - the rear angle of the cutting wedge of the wormtype tool.

To calculate the coordinates of the node points of the axial section of the worm-type tool, it is necessary to make a transition from the coordinate system $X_{2}^{\prime} Y_{2}^{\prime} Z_{2}^{\prime}$ to the system $X_{2} Y_{2} Z_{2}$. We make this transition in three steps (Fig. 9):

a) rotate the coordinate system $X_{2}^{\prime} Y_{2}^{\prime} Z_{2}^{\prime}$ around the axis $X_{2}^{\prime}$ by an angle equal to $\pi / 2-\lambda_{2}$, then the coordinate system $X_{2}^{\prime} Y_{2}^{\prime} Z_{2}^{\prime}$ will take a position $X_{26}^{\prime} Y_{2 \beta}^{\prime} Z_{26}^{\prime}$, while the axis $Z_{2 \mathrm{~B}}^{\prime}$ is parallel $Z_{2}$;

b) we transfer the origin of coordinates $O_{2}^{\prime}$ in $O_{2}$ due to its displacement in the direction of the axis $X_{2 \beta}^{\prime}$ by an amount $r_{a 2}$ and in the direction of the axis $Z_{2 \beta}^{\prime}$ by the value of the segment $M N=p_{2} \vartheta_{2}$, where $p_{2}$ - the parameter of the screw cutting edge. In this case, the system $X_{2 \beta}^{\prime} Y_{2 \beta}^{\prime} Z_{2 \beta}^{\prime}$ will move to the coordinate system $X_{2 \beta} Y_{2 \beta} Z_{2 \beta}$; 
c) rotate the coordinate system $X_{2 \beta} Y_{2 \beta} Z_{2 \beta}$ around the axis $Z_{2}$ by an angle $\vartheta_{2}$, then the coordinate system $X_{26} Y_{2 \beta} Z_{2 \beta}$ will take the position $X_{2} Y_{2} Z_{2}$.

Conversion of coordinates is realized by means of the following matrix equality:

$$
r_{2}=M_{22 \beta} M_{262 \beta^{\prime}} M_{26^{\prime} 2^{\prime}} r_{2^{\prime}},
$$

Where $r_{2}$ and $r_{2^{\prime}}$ - the column matrices of the corresponding radii of the vectors of the same point in the systems $X_{2} Y_{2} Z_{2}$ and $X_{2}^{\prime} Y_{2}^{\prime} Z_{2}^{\prime}$;

$M_{26^{\prime} 2^{\prime}}$ - matrix of transition from system $X_{2}^{\prime} Y_{2}^{\prime} Z_{2}^{\prime}$ to system $X_{2 \beta}^{\prime} Y_{2 \beta}^{\prime} Z_{2 \beta}^{\prime}$;

$M_{2626^{\prime}}$ - matrix of transition from system $X_{2 \beta}^{\prime} Y_{2 \beta}^{\prime} Z_{2 \beta}^{\prime}$ to system $X_{2 \beta} Y_{2 \beta} Z_{2 \beta}$;

$M_{22 \beta}$ - matrix of transition from system $X_{2 \beta} Y_{2 \beta} Z_{2 \beta}$ to system $X_{2} Y_{2} Z_{2}$.

In the coordinate form, taking into account expression (3), the transition from the coordinate system $X_{2}^{\prime} Y_{2}^{\prime} Z_{2}^{\prime}$ to the coordinate system $X_{2} Y_{2} Z_{2}$ will be determined by equations that can be written:

$$
\begin{gathered}
x_{2}=\left(x_{2}^{\prime}+r_{a 2}\right) \cos \vartheta_{2}-\left(y_{2}^{\prime} \sin \lambda_{2}+z_{2}^{\prime} \cos \lambda_{2}\right) \sin \vartheta_{2} \\
y_{2}=\left(x_{2}^{\prime}+r_{a 2}\right) \sin \vartheta_{2}+\left(y_{2}^{\prime} \sin \lambda_{2}+z_{2}^{\prime} \cos \lambda_{2}\right) \cos \vartheta_{2}(4) \\
z_{2}=-y_{2}^{\prime} \cos \lambda_{2}+z_{2}^{\prime} \sin \lambda_{2}+p_{2} \vartheta_{2}
\end{gathered}
$$

Equating the expression $x_{2}$ for zero, we obtain the equation:

$$
\left(x_{2}^{\prime}+r_{a 2}\right) \cos \vartheta_{2}-\left(y_{2}^{\prime} \sin \lambda_{2}+z_{2}^{\prime} \cos \lambda_{2}\right) \sin \vartheta_{2}=0
$$

Substituting for this equation the expressions for $x_{2}^{\prime}$, $y_{2}^{\prime}$ and $z_{2}^{\prime}$ from (2) after a simple transformation we obtain the equation from which we can obtain the formula for calculating the angle $\vartheta_{2}$ :

$$
\vartheta_{2}=\operatorname{arctg}\left(\frac{q_{2} \cos \alpha_{2}-r_{a 2}}{q_{2} \sin \alpha_{2} \sin \lambda_{2}}\right) .
$$

If, for a fixed value $q_{2}$, we calculate $\vartheta_{2}$ by formula (6) and substitute the obtained value $\vartheta_{2}$ into expressions (4) for $y_{2}$ and $z_{2}$, then it is possible to determine the coordinates of the node point of the axial section of the lateral surface of the screw of the worm-type tool.

The obtained coordinates of the node points of the axial section of the lateral surface of the turn of the worm-type tool allow to determine the parameters for setting the grinding wheel with respect to the worm-type tool. These adjusting parameters will make it possible to carry out the most labor-intensive operation of manufacturing this tool, namely, grinding the lateral surface of the winding.

\section{References}

1. A.A. Matalin, Manufacturing engineering (Lan, St. Petersburg, 2010)

2. M.F. Pashkevich, Manufacturing engineering. Course and degree design (Grevtsov's publishing house, Minsk, 2010)

3. V.L. Kulygin, Manufacturing engineering (BASTET, Moscow, 2011)

4. O.V. Taratynov, Manufacturing engineering. Design bases on the computer (FORUM, Moscow, 2011)

5. I.S. Ivanov, Manufacturing engineering (INFRA-M, Moscow, 2012)

6. 6. V.A. Arshinov, G.A. Alekseev, Cutting of metals and the cutting tool (Mechanical engineering, Moscow, 1968)

7. P.R. Rodin, Metal-cutting tools (Vishcha shkola, Kiev, 1974)

8. I.A. Ordinartsev, Reference book of the toolmaker (Mechanical engineering, Leningrad, 1987)

9. G.N. Sakharov, O.B. Arbuzov, Yu.L. Borovoy, Metal-cutting tools (Mechanical engineering, Moscow, 1989)

10. O.I. Averyanov, V.V. Klepikov, Cutting tool (MGIU, Moscow, 2007)

11. A.A. Ryzhkin, K.G. Shuchev, A.G. Skhirtladze, Cutting tool (Phoenix, Rostov-on-Don, 2009)

12. E.E. Feldstein, M.A. Korniyevich, Cutting tool. Operation (INFRA-M, Moscow, 2012)

13. D.V. Kozhevnikov, V.A. Grechishnikov, S.V. Kirsanov, S.N. Grigoriev, Cutting tool (Mechanical engineering, Moscow, 2014)

14. Inventor's certificate USSR, no. 1106609, 1984

15. Inventor's certificate USSR, no. 1004030, 1983

16. Patent RF, no. 103771, 2011

17. Patent RF, no. 2005013, 1993

18. N.V. Smorkalov, Numerical modeling of surfaces when processing by cutting (Publishing house of the Kama state polytechnical institute, Naberezhnye Chelny, 2003) 